\title{
Apocalipsis cosmológico: mitos y metáforas del fin en la astronomía contemporánea ${ }^{1}$
}

\section{Cosmological Apocalypse: Myths and Metaphors of the End in Contemporary Astronomy}

\author{
Alberto Fragio \\ Universidad Autónoma Metropolitana de México D.F. - Unidad Cuajimalpa \\ afragio@correo.cua.uam.mx
}

Recibido: 18/01/2015

Aceptado: 24/06/2015

\section{Resumen}

Los hallazgos en astronomía observacional y en astrofísica extragaláctica producidos durante los siglos Xx y XXI, así como la subsiguiente multiplicación de especialidades y subespecialidades que componen en la actualidad la ciencia astronómica - como la radioastronomía, la geología planetaria, la astronometría o la astronomía de rayos X, entre otras muchas-, no sólo han permitido la pervivencia del mito sino que han propiciado su secreta renovación. Con sus sensacionales descubrimientos, la astronomía contemporánea ha remitificado el cosmos, produciendo de paso nuevos mitos del origen y del final del universo. En este artículo proponemos que tanto la cosmología escatológica como el apocalipsis cosmológico habrían practicado una reocupación (astronómica) del mito y de la metáfora.

Palabras clave: Albert Einstein, Alexander Friedmann, George Lemaître, reocupación cosmológica de la escatología, Hans Blumenberg.

\footnotetext{
${ }^{1}$ La redacción de este pequeño texto ha sido posible gracias a una beca postdoctoral concedida por la Gerda Henkel Stiftung, disfrutada en el Institut für Medizingeschichte und Wissenschaftsforschung der Universität zu Lübeck. También se ha beneficiado del proyecto de investigacion "Elementos subjetivos y objetivos en la historia cultutal del bienestar (Well-being): 1750-1950. Epistemologia historica y economia moral" (FFI2013-46361-R), financiado por el Ministerio de Economía y Competitividad del Gobierno de España; y del programa mexicano de la DSA-SEP “Apoyo a la incorporación de nuevos Profesores de Tiempo Completo” (UAM-PTC-521, 2015-2016).
} 


\begin{abstract}
Discoveries in observational astronomy and extragalactic astrophysics made during the $\mathrm{xx}^{\mathrm{th}}$ and $\mathrm{xxI}^{\mathrm{st}}$ centuries and the subsequent proliferation of the specialties and subspecialties found in contemporary astronomy -such as radio astronomy, planetary geology, astronometry or X-ray astronomy, among many others- have allowed both the survival of the myth and its continual renewal. The sensational discoveries made by contemporary astronomy has re-mythologized the cosmos, producing new myths both of the origins of the Universe and it's end while remaining consistent with the cosmological tradition within which it falls. In this paper, I suggest that both eschatological cosmology and the cosmological apocalypse have introduced an (astronomical) reoccupation of myth and metaphor.

Keywords: Albert Einstein, Alexander Friedmann, George Lemaître, cosmological reoccupation of eschatology, Hans Blumenberg.
\end{abstract}

"Einstein hablaba con tanta frecuencia de Dios que casi podemos considerarlo un teólogo enmascarado".

Friedrich Dürrenmatt².

"He recibido su carta del 10 de junio. Nunca he hablado con un sacerdote jesuita en mi vida, y estoy asombrado por la audacia de tales mentiras sobre mí. Desde el punto de vista de un sacerdote jesuita, soy, por supuesto, y he sido siempre, un ateo". Carta de Einstein a Guy H. Raner, fechada el 2 de julio de $1945^{3}$.

\title{
1. Introducción
}

Con motivo del 70 cumpleaños de Albert Einstein [1879-1955], Paul A. Schlipp tuvo la iniciativa de editar y publicar un volumen en la colección The Library of Living Philosophers, bajo el título Albert Einstein: Philosopher-Scientist [1949] . En este volumen se daban cita algunos de los físicos y pensadores más prominentes de la época, entre ellos el astrofísico y sacerdote belga Georges Lemaître, quien algún tiempo después habría de valorar este monográfico como una de las obras más

\footnotetext{
${ }^{2}$ F. Dürrenmatt, Albert Einstein, Zürich, Diogenes Verlag, 1979, p. 12, citado por M. Jammer, Einstein und die Religion, Konstanz, Universitätsverlag Konstanz, 1995, p. 54: "Einstein pflegte so oft von Gott zu reden, dass ich beinahe vermute, er sei ein verkappter Theologe gewesen".

${ }^{3}$ Citado por M. R. Gilmore, “Einstein's God. Just What Did Einstein Believe About God?" en Skeptic Magazine, "The God Question", 5, n. ${ }^{\circ}$ 2, 1997, pp. 62 y ss.

${ }^{4}$ P. A. Schlipp (ed.), Albert Einstein: Philosopher-Scientist [1949], The Library of Living Philosophers, volume VII, Carbondale, Illinois, Sothern Illinois University (3rd ed. 1995).
} 
importantes de la historia de la ciencia ${ }^{5}$. Las "Notas autobiográficas" $"$ que Einstein escribió tres años antes para el libro de Schlipp comenzaban con estas dramáticas palabras: "Aquí me encuentro, a mis sesenta y siete años, preparado para escribir algo así como mi propia necrología $[\ldots]$ ". . Para entonces hacía ya mucho que Einstein había desistido de defender un modelo de universo estático, abiertamente incompatible con cualquier comprensión escatológica del cosmos, si bien aún mantenía vivo un "sentimiento religioso cósmico", tal como lo describiera en otra ocasión. A este sentimiento religioso se había referido como "el motivo más fuerte y más noble de la investigación científica: sólo quienes entienden los inmensos esfuerzos y, sobre todo, esa devoción sin la cual sería imposible el trabajo innovador en la ciencia teórica, son capaces de captar la fuerza de la única emoción de la que puede surgir tal empresa, siendo como es algo alejado de las realidades inmediatas de la vida". Desde este punto de vista, "las únicas personas profundamente religiosas son los investigadores científicos serios" ${ }^{\prime 10}$. En unos términos algo más matizados, no obstante, hubo de expresarse al comienzo de sus "Notas autobiográficas", en un pasaje que sin duda merece citarse en toda su extensión:

"Muy pronto tomé conciencia de la insignificancia de las aspiraciones e ilusiones
que abruman sin descanso a la mayoría de los hombres durante toda su vida. Tam-
poco tardé en ver qué cruel era esa inquietud, cuya dureza entonces se disfrazaba de
hipocresía y grandes palabras mejor que hoy. La necesidad condenaba a participar
en esa carrera por la supervivencia, pero aunque resolviera sus necesidades físicas,
no satisfacía al hombre como ser que piensa y siente. La religión era la primera op-
ción que el sistema educativo ofrecía a los niños. Así, aunque mis padres eran judíos
absolutamente descreídos, yo fui profundamente religioso hasta que cumplí doce
años. Los libros de divulgación científica que leía me demostraron que los relatos
bíblicos no podían ser ciertos y, consecuentemente, terminé siendo un librepensador

${ }^{5}$ G. Lemaître, "Rencontres avec A. Einstein", en Revue des Questions Scientifiques 129, n. ${ }^{1}$, 1958, pp. 129-32, citado por K. Roessler, "Georges Lemaître, das expandierende Universum und die kosmologische Konstante", en H. W. Duerbeck y W. R. Dick (eds.), Einsteins Kosmos. Untersuchungen zur Geschichte der Kosmologie, Relativitätstheorie und zu Einsteins Wirken und Nachwirken, Frankfurt am Main, Verlag Harri Deutsch, 2005, pp. 178-9.

${ }^{6}$ A. Einstein, "Notas autobiográficas" [1949], trad. esp. de A. Castelar, en J. M. Sánchez Ron (ed.), Albert Einstein. Obra esencial, Barcelona, Crítica, 2005, pp. 43-83.

${ }^{7}$ Ibidem, p. 44. Como indicó J. Merleau-Ponty en su monografía sobre Einstein, no era ésta precisamente una coquetería. Véase su Einstein, Paris, Flammarion, 1993, p. 109.

${ }^{8}$ A. Einstein, "Religión y ciencia" [1930], trad. de A. Goldar, en J. M. Sánchez Ron (ed.), Albert Einstein. Obra esencial, op. cit., p. 292. La literatura especializada sobre Einstein y la religión es muy abundante. En lengua castellana puede verse H. H. Benítez, Einstein y la religión. Relaciones entre ciencia y creencia, Santiago de Chile, RIL editores, 2007.

${ }^{9}$ A. Einstein, "Religión y ciencia", op. cit., p. 292.

${ }^{10}$ Ibidem. 
fanático [...]. La impresión de aquellos años derivó en una desconfianza hacia toda autoridad, en un escepticismo hacia las creencias de cualquier sociedad, actitud que jamás abandoné, si bien más tarde, cuando alcancé una mejor comprensión de las realidades causales, se moderó.

Ahora sé que la pérdida de aquel paraíso religioso de la infancia fue mi primer intento de liberarme de las ataduras 'meramente personales' de una vida dominada por sueños, anhelos y sentimientos primarios. Más allá e independientemente de los hombres, se extendía el gran mundo que se alza ante nosotros misterioso, enorme y eterno, pero comprensible, al menos parcialmente, mediante la investigación y el pensamiento. Contemplarlo parecía liberar de esas ataduras y pronto sentí que más de un hombre a quien yo apreciaba y admiraba había alcanzado la libertad y la paz interior dedicándose a ello con devoción. De manera consciente o inconsciente, la comprensión de ese mundo sirviéndome de mis capacidades pasó a ser mi meta suprema. Decidí seguir a aquellos hombres que, animados por las mismas motivaciones, en el pasado y en el presente, habían comenzado a comprenderlo. Aunque el camino no era tan fácil ni tan atractivo como el paraíso religioso, ha demostrado ser tan sólido que jamás me he arrepentido de haberlo tomado""1.

Cuando un rabino neoyorkino llamado Herbert S. Goldstein le envió un telegrama a Einstein para preguntarle si creía en Dios, Einstein respondió de la forma más breve: "Yo creo en el Dios de Spinoza, que se revela en la armonía natural de los seres, no en un Dios que se apropia de los destinos y acciones humanas" 12 . En este sentido, la apelación al misterio del mundo en las "Notas autobiográficas" podemos entenderla como una forma peculiar de religiosidad -después de todo-, de religiosidad cósmica $^{13}$, que se especificaría en la apertura a la transcendencia objetiva del universo: un auténtico punto de fuga tanto de la metafísica como del mundo de la vida. Una forma de religiosidad semejante, emparentada con el Deus sive natura spinocista $^{14}$, no sólo había de eliminar de raíz el topos de la salvación personal, sino

\footnotetext{
${ }^{11}$ A. Einstein, "Notas autobiográficas”, op. cit., pp. 43-4. Einstein añadía: “[...] La variedad de las circunstancias externas y los momentos concretos implican una especie de atomización de la vida de cada uno. El giro decisivo en un hombre de mi talante se produce cuando la atención se separa progresivamente de lo momentáneo y de lo meramente personal y se centra en la pretensión de aprehender conceptualmente las cosas". Ibidem, pp. 44-5. Para un análisis del "talante" de Einstein se puede ver L. Daston, "A Short History of Einstein's Paradise beyond the Personal", en P. Galison, G. Holton y S. S. Schweber (eds.), Einstein for the $21^{\text {st }}$ Century, New Jersey, Princeton University Press, 2008, pp. 15-26. ${ }^{12}$ Citado por M. Jammer, Einstein und die Religion, op. cit., p. 31: "Ich glaube an Spinozas Gott, der sich in der gesetzlichen Harmonie des Seienden offenbart, nicht an einen Gott, der sich mit den Schicksalen und Handlungen der Menschen abgibt".

${ }^{13}$ Ibidem, pp. 44-5.

${ }^{14}$ M. Jammer, Einstein und die Religion, op. cit., p. 31; y Jürgen Audretsch, "Vorwort”, en Max Jammer, ibidem., p. 8. Para el transfondo teológico y religioso de la cosmología moderna, véanse los libros de H. Kragh, Matter and Spirit in the Universe: Preludes to Modern Cosmology, London, Imperial College
} 
dar por descontados la eternidad e inmutabilidad del cosmos: "el gran mundo" independiente de los hombres. No en vano, se ha conjeturado que el influjo de la teología natural de Spinoza pudo haber sido determinante en la temprana aceptación, por parte de Einstein, de las soluciones estáticas en la cosmología relativista ${ }^{15}$.

A nuestro juicio, estos presupuestos teológicos tienen no poca relevancia a la hora de explicar la vehemencia con la que Einstein hubo de rechazar en un primer momento las soluciones no estáticas ${ }^{16}$ propuestas de manera independiente por el matemático y meteorólogo ruso Alexander Friedmann [1888-1925] y el astrofísico y sacerdote belga Georges Lemaître [1894-1966], en 1922-1923 y en 1927, respectivamente ${ }^{17}$. En último término, los modelos no estáticos de universo conllevaban un "exceso" de misterio inasumible en el apacible panteísmo cósmico defendido por Einstein.

Si bien "la más profunda y maravillosa experiencia que puede tener un hombre es el sentido del misterio"18, las implicaciones escatológicas de los modelos de Fried-

Press, 2004; y Entropic Creation. Religious Contexts of Thermodynamics and Cosmology, Aldershot, Ashgate, 2008.

${ }^{15}$ M. Jammer, Einstein und die Religion, op. cit., p. 38. Una apretada síntesis de la historia de la cosmología relativista en L. E. Otero, "La cosmología relativista: del universo infinito y estático al universo en expansión", en Umbral. Revista de la Facultad de Estudios Generales de la Universidad de Puerto Rico, 2008, pp. 1-34.

16 "Einstein planteó una serie de ecuaciones tensoriales que describían la curvatura del espacio-tiempo en función de la intensidad de los campos gravitatorios. Según el tipo de soluciones que se obtuvieran, las aplicaciones cosmológicas de dichas ecuaciones serían unas u otras, o dicho de otro modo, el universo teórico resultante tendría unas características u otras". A. Rioja y J. Ordóñez, Teorías del universo, Madrid, Editorial Síntesis, vol. 3, 2006, p. 335.

${ }^{17}$ En esta nómina habría que incluir también a Willem de Sitter: “[...] en el mismo año en que aparecieron las 'Consideraciones cosmológicas', un astrónomo holandés amigo de Einstein [...] publicaba un escrito en las actas de la Academia de Ciencias de Amsterdam (concretamente el 31 de marzo de 1917), en el que sometía a discusión el resultado obtenido por su ilustre amigo. Profesor de Astronomía Teórica de la Universidad de Leiden y posteriormente director del observatorio astronómico de dicha ciudad, De Sitter ponía de manifiesto que la solución hallada por Einstein a las ecuaciones de campo no era la única posible (preservando la constante cosmológica $\lambda$ ). Y puesto que el modelo teórico de universo resultante dependía del tipo de solución planteada, quería decirse que, partiendo de las mismas ecuaciones, al menos más de un modelo de universo era posible. En el caso del denominado 'modelo de Sitter', nos enfrentamos a un universo, o si se quiere, a un espacio-tiempo 'vacío' de materia, por oposición al universo 'lleno' (aunque muy poco denso) de Einstein. Se trata de una construcción puramente matemática, en la que se pone de manifiesto que la introducción de partículas materiales de prueba daría lugar a un proceso de alejamiento de unas con respecto a otras. De Sitter deducía así la expansión del universo a partir de la teoría general de la relatividad [...]". A. Rioja y J. Ordóñez, op. cit., pp. 338-9. ${ }^{18}$ A. Einstein, "Mi credo", discurso pronunciado ante la Liga Germana de Derechos Humanos, en Berlín en el otoño de 1932, recogido en M. White y J. Gribbin, Einstein, a Life in Science, London, Simon \& Schuster LTD., 1993, p. 262. De este modo continúa la cita: "Aquel que nunca haya tenido esta experiencia me parece, si no muerto, al menos ciego. Sentir que detrás de cualquier cosa que se pueda experimentar existe algo que nuestra mente no puede abarcar y cuya belleza y sublimidad nos alcanza sólo indirectamente como un débil reflejo, esto es religiosidad. En este sentido sí soy religioso. Para mí 
mann y Lemaître forzosamente habían de resultarle intolerables a Einstein. Pero a pesar de todas sus reticencias, de la peculiar mística desmundanizadora de la que hacía gala ${ }^{19}$ - y de la comprensión griega clásica del cosmos a ella asociada ${ }^{20}-$, Einstein no pudo evitar el destino que Friedmann y Lemaître habían preparado para su cosmología relativista: convertirla en una cosmología escatológica. Es por ello que no debe extrañarnos que la propia cosmología física contemporánea incluya a su vez una comprensión escatológica del universo. La paradoja histórica consistió en que el arranque de la cosmología científica a comienzos del siglo $\mathrm{xx}^{21}$ hubo de coincidir con la reocupación cosmológica de la escatología.

\section{El universo como gas de estrellas: el primer modelo cosmológico de Einstein}

Como es conocido, el momento fundacional de la cosmología relativista se fecha en 1917, cuando Einstein publicó sus "Kosmologische[n] Betrachtungen zur allgemeinen Relativitätstheorie" 22 en la Real Academia Prusiana de Ciencias de Berlín ${ }^{23}$. En este famoso artículo, Einstein propuso un modelo de universo estático, espacialmente cerrado y caracterizado por una distribución uniforme de materia ${ }^{24}$; es lo que se ha venido a denominar el modelo estático de la cosmología relativista clásica $^{25}$. En este primer modelo, Einstein aplicaba la teoría general de la relatividad al universo en su conjunto, haciendo uso de la geometría riemanniana para describir

es suficiente con maravillarme con estos secretos e intentar humildemente de hacer en mi mente una imagen de la elevada estructura de todo lo que existe".

${ }^{19}$ M. Jammer, Einstein und die Religion, op.cit., p. 53.

${ }^{20}$ Véase A. Fragio, "La ontología cosmológica en la obra temprana de Hans Blumenberg: las Beiträge y Die ontologische Distanz", en Res publica 23, Murcia, 2010, pp. 93-122; A. Fragio, "Astronomía, cosmogonía y hermenéutica de la Modernidad: el debate entre Hans Blumenberg y Carl Friedrich von Weizsäcker", en José Luis Villacañas (ed.) Hans Blumenberg: la apuesta por la Ilustración tardía. Revista Anthropos. Huellas del conocimiento 238, 2013. pp. 43-55; A. Fragio, "La destrucción de las comprensiones teológicas de la Modernidad", en Endoxa 26, 2010, pp. 243-78.

${ }^{21}$ Una buena reconstrucción de este periodo sigue siendo la de J. Merleau-Ponty, Cosmologie du XX siècle. Étude épistémologique et historique des théories de la cosmologie contemporaine, Paris, Éditions Gallimard, 1965, pp. 35-108. Véase asimismo el magnífico trabajo de M. Longair, The Cosmic Century. A History of Astrophysics and Cosmology, Cambridge, Cambridge University Press, 2006.

${ }^{22}$ A. Einstein, "Kosmologische[n] Betrachtungen zur allgemeinen Relativitätstheorie", Sitzungsberichte der Königlich Preußischen Akademie der Wissenschaften zu Berlin, 1917, pp. 142-52. Trad. esp. de J. García Sanz, "Consideraciones cosmológicas sobre la teoría de la relatividad general", en J. M. Sánchez Ron (ed.), Albert Einstein. Obra esencial, op.cit, pp. 494-506.

${ }^{23}$ Una revisión de las aportaciones de Einstein a la cosmología puede verse en T. Jung, "Einsteins Beitrag zur Kosmologie - ein Überblick", en H. W. Duerbeck y W. R. Dick (eds.), Einsteins Kosmos. Untersuchungen zur Geschichte der Kosmologie, Relativitätstheorie und zu Einsteins Wirken und Nachwirken, Frankfurt am Main, Verlag Harri Deutsch, 2005, pp. 66-107.

${ }^{24}$ Ibidem, p. 68.

${ }^{25}$ E. Scholz, "Einstein-Weyl Models of Cosmology", en J. Renn (ed.), Albert Einstein. Chief Engineer of the Universe. One Hundred Authors for Einstein, Berlin, WILEY-VCH, 2005, p. 395. 
la métrica del espacio-tiempo, y mostrando así la inextricable relación entre la gravedad y las propiedades estructurales del universo a gran escala ${ }^{26}$. En palabras del propio Einstein: "según la teoría general de la relatividad, el carácter métrico (curvatura) del continuo espacio-temporal tetradimensional está definido en cada punto por la materia en dicho punto y el estado de dicha materia". ${ }^{27}$ En consecuencia, "la curvatura del espacio es variable en el tiempo y el lugar, según la distribución de materia $[\ldots]^{\prime 28}$.

La metáfora cosmológica de referencia de este modelo de universo - de gran valor heurístico- era el gas de estrellas ${ }^{29}$. Einstein consideraba el universo como un gas en equilibrio, sometido a la ley de distribución de Boltzmann ${ }^{30}$. Pero debido a la falta de uniformidad en la distribución de las estrellas -la materia del universo, en este modelo- la estructura métrica debía ser "por fuerza extraordinariamente complicada. Pero si estamos interesados sólo en la estructura a gran escala, podemos representarnos la materia como estando uniformemente distribuida sobre espacios enormes, de modo que su densidad de distribución es una función variable, que varía de forma extraordinariamente lenta" ${ }^{\prime 3}$.

Así planteadas las cosas, el universo en su totalidad podía ser "groseramente aproximado por medio de un espacio esférico" 32 . Este espacio esférico poseería un radio fijo y estaría uniformemente rellenado con el gas estelar a la manera de un fluido ideal de masa y densidad constantes ${ }^{33}$. Sin embargo, para que esta suerte de fluido cósmico permaneciera en equilibrio gravitatorio, Einstein se vio obligado a introducir en las ecuaciones relativistas una fuerza ad hoc, de carácter repulsivo, que compensaba la atracción gravitatoria entre las masas puntuales del gas de estrellas, la denominada "constante cosmológica". Dado que en este modelo la materia se encontraba distribuida de manera discreta y poseía movimientos peculiares desordenados, en ausencia de una fuerza que compensase exactamente la densidad de energía de las masas estelares en reposo, se habría de producir una expansión o contracción

\footnotetext{
${ }^{26}$ Para más detalles puede verse J.-P. Luminet, L'invention du Big Bang [1997], Paris, Éditions du Seuil, 2004, pp. 31 y ss.

${ }^{27}$ A. Einstein, "Consideraciones cosmológicas sobre la teoría de la relatividad general", op. cit., p. 501.

${ }^{28}$ Ibidem, p. 506.

${ }^{29}$ J.-P. Luminet, L'invention du Big Bang, op. cit., pp. 32-3.

${ }^{30}$ A. Einstein, "Consideraciones cosmológicas", op. cit., p. 496.

${ }^{31}$ Ibidem, p. 501.

${ }^{32}$ Ibidem, p. 506. Podemos ubicar esta aseveración en la tradición kepleriana que despojaba al cosmos de sus características geométricas como expresión de su perfección.

${ }^{33}$ E. Scholz, "The Standard Model of Contemporary Cosmology", en J. Renn (ed.), Albert Einstein. Chief Engineer of the Universe, op. cit., p. 388.
} 
crecientes $^{34} \mathrm{y}$, por ende, la métrica del universo se volvería variable en el tiempo ${ }^{35}$. Sin constante cosmológica cualquier pequeña variación de la densidad de materia produciría una evolución irreversible del cosmos: o bien comenzaría a expandirse, o bien a contraerse ${ }^{36}$. Es decir: el universo tendría un principio y un final, adquiriría una historia.

Muchos años después de proponer su primer modelo cosmológico, Einstein hubo de justificar sus primeros planteamientos destacando que "no había razón alguna por la que dudar de la naturaleza estática del espacio" ${ }^{37}$. Por más arbitraria que ahora nos pueda parecer la introducción de la constante cosmológica, conviene recordar que en la época en la que Einstein propuso su modelo de universo, nada se sabía de objetos cosmológicos espectaculares como estrellas supermasivas, agujeros negros, núcleos galácticos o cuásares ${ }^{38}$. De hecho ni siquiera estaba establecido que hubiese otras galaxias o cuerpos extragalácticos de ningún tipo ${ }^{39}$ : "en estas circunstancias, y dada la escasez de datos, era natural suponer a priori un universo estático" ${ }^{40}$. No obstante, el propio Einstein había cerrado con prudencia sus "Kosmologische[n] Betrachtungen" señalando que si bien su modelo era "lógicamente consistente" y el "más accesible" para la teoría general de la relatividad, no creía conveniente entrar a valorar si acaso era también "sostenible desde el punto de vista del conocimiento astronómico presente" ${ }^{" 41}$.

\footnotetext{
${ }^{34}$ J. Cepa, Cosmología física, Barcelona, Ediciones Akal, 2007, p. 155. Cepa nos recuerda que, “[...] ya Newton hizo notar que si el universo no estuviera uniformemente lleno de estrellas, se colapsaría debido a la atracción gravitatoria, empezando por las partes más densas". Ibidem, p. 15. Véase también A. Rioja y J. Ordóñez, Teorías del universo, op. cit., pp. 316-7 y p. 336: "desde hacía tiempo se sabía que todo intento de extrapolar [la teoría newtoniana de la gravitación] se enfretaba con la siguiente dificultad: o toda la materia existente se concentra en una región finita del espacio infinito, en cuyo caso el sistema es inestable, o la materia está uniformemente distribuida en el espacio hasta el infinito, en cuyo caso el campo de gravitación debe ser infinito en cada punto". Para la historia de la paradoja cosmológica de la gravedad en la mecánica newtoniana véase B. Suchan, Die Stabilität der Welt. Eine Wissenschaftsphilosophie der kosmologischen Konstante, Paderborn, Mentis, 1999, pp. 28-44.

${ }^{35}$ J.-P. Luminet, L'invention du Big Bang, [1997], Paris, Éditions du Seuil, 2004, pp. 33-4; J.-P. Luminet, Le Destin de l'Univers. Trous noirs et énergie sombre [2006], tome II, Paris, Gallimard, 2010, pp. 798-9.

${ }^{36}$ M. V. Sazhin, Cosmología moderna [2002], trad. esp. A. L. Malca, Moscú, Editorial URSS, 2005, p. 69.

${ }^{37}$ K. Roessler, "Georges Lemaître, das expandierende Universum und die kosmologische Konstante", en H. W. Duerbeck y W. R. Dick (eds.), Einsteins Kosmos, op. cit., pp. 177-8.

${ }^{38}$ M. Schemmel, "Gekrümmte Universen von Einstein: Karl Schwarzschilds kosmologische Spekulationen und die Anfänge der relativischen Kosmologie", en H. W. Duerbeck y W. R. Dick (eds.), Einsteins Kosmos, op. cit., p. 58.

${ }^{39}$ J. Cepa, Cosmología fisica, op. cit., p. 154.

${ }^{40}$ Ibidem.

${ }^{41}$ A. Einstein, "Consideraciones cosmológicas sobre la teoría de la relatividad general”, op. cit., p. 506.
} 


\section{La reocupación cosmológica de la escatología: los modelos no estáticos de universo}

La deriva escatológica de la cosmología relativista clásica era en cierto modo inevitable, y los esfuerzos de Einstein por preservar la inmutabilidad del universo se demostraron inútiles. Si la teoría general de la relatividad permitía que la curvatura del espacio variase localmente con el tiempo, parecía razonable suponer que la métrica del universo en su conjunto también pudiera cambiar, dando lugar a fenómenos de contracción o dilatación del medio cósmico ${ }^{42}$. Como ya hemos mencionado, Alexander Friedmann y Georges Lemaître no tardaron en proponer soluciones no estáticas a las ecuaciones de Einstein, que arrojaban el resultado de un modelo dinámico de universo. La cosmología relativista pasaba así a convertirse en una cosmología evolutiva ${ }^{43}$, en la que el universo entero habría de desplegar sus acontecimientos en una temporalidad histórica lineal o, eventualmente, cíclica. El universo adquiría una historia y, con ella, una escatología.

A nuestro modo de ver, el lugar en el que se operó esta reocupación cosmológica de la escatología fue en el debate sobre la constante cosmológica ${ }^{44}$, el misterio por excelencia. Al preservar la estaticidad del mundo, la constante cosmológica había impedido una comprensión escatológica del universo, alejando las categorías de inicio y fin, y perpetuando la comprensión griega clásica del cosmos y sus prerrogativas de eternidad, racionalidad y seguridad. De esta manera no sólo había quedado garantizada la transcendencia objetiva del universo, sino que se eliminaba de raíz cualquier concepción mesiánica o apocalíptica del tiempo cósmico.

Pero cuando Alexander Friedmann halló las soluciones dinámicas de las ecuaciones de Einstein, la escatología se instaló en la cosmología, adquiriendo de paso un sólido respaldo matemático. En 1922 Friedmann publicó en la revista alemana Zeitschrift für Physik el célebre artículo "Über die Krümmung des Raumes" ["Sobre la curvatura del espacio"], en donde afirmaba que la métrica del universo era susceptible de variar en el tiempo ${ }^{45}$. Las ecuaciones propuestas por Friedmann prescindían de hecho de la constante cosmológica, y predecían que el universo

\footnotetext{
${ }^{42}$ J.-P. Luminet, Le Destin de l'Univers, tome II, op. cit., pp. 781-2.

${ }^{43}$ Ibidem, p. 775. Véase también K. Roessler, "Georges Lemaître, das expandierende Universum und die kosmologische Konstante", en H. W. Duerbeck y W. R. Dick (eds.), Einsteins Kosmos, op. cit., p. 162

${ }^{44}$ Para un estudio pormenorizado de la constante cosmológica véase B. Suchan, Die Stabilität der Welt. Eine Wissenschaftsphilosophie der kosmologischen Konstante, op. cit., pp. 103-34. El lector interesado también podrá encontrar una breve reconstrucción histórica en M. V. Sazhin, Cosmología moderna, op. cit., pp. 69 y ss.

${ }^{45}$ Dos años después, en 1924, publicó en esa misma revista el no menos célebre "Über die Möglichkeit einer Welt mit konstander negativer Krümmung" ["Sobre la posibilidad de un universo con curvatura negativa constante"], en Zeitschrift für Physik 21, 1924, p. 326.
} 
no será, en general, estático ${ }^{46}$. Si bien era cierto que no había causa alguna por la cual el universo debiera expandirse o contraerse, una vez que empezara a variar su métrica, no cesaría de hacerlo.

En El mundo como espacio y tiempo [1923], Friedmann definía dos tipos de universo: el universo estacionario y el universo variable ${ }^{47}$. En el primero de ellos la curvatura del espacio no se modifica en el transcurso del tiempo, mientras que en el segundo sí lo hace: "como ilustración del primer tipo de universo puede servir una esfera cuyo radio no varía con el tiempo; la superficie bidimensional de esta esfera será precisamente un espacio bidimensional de curvatura constante. Por el contrario, el segundo tipo de universo puede ser representado mediante una esfera que cambia continuamente, bien expandiéndose, bien disminuyendo su radio como si se comprimiera" $"$.

Einstein no aceptó en modo alguno las soluciones de Friedmann ${ }^{49}$, que consideró erróneas y que pronto cayeron en el olvido. Pero fue Georges Lemaître ${ }^{50}$ quien unos pocos años después reintroduciría la constante cosmológica en un nuevo modelo dinámico de universo, y ofrecería una interpretación cosmológica consistente de los hallazgos observacionales de Vesto Slipher [1875-1969] y Edwin Powell Hubble [1889-1953] sobre los desplazamientos al rojo ${ }^{51}$ de las nebulosas

\footnotetext{
${ }^{46}$ J. Cepa, Cosmología fisica, op. cit., p. 134; J.-P. Luminet, Le Destin de l'Univers, tome II, op. cit., p. 799.

${ }^{47}$ A. A. Friedmann, El mundo como espacio y tiempo [1923], trad. esp. de G. Peña, Moscú, Editorial URSS, 2003, pp. 196 y ss.

${ }^{48}$ Ibidem, p. 197.

${ }^{49}$ Para los detalles sobre la recepción de Einstein de los trabajos de Friedmann véase J.-P. Luminet, Le Destin de l'Univers, op. cit. cap. 5, pp. 47-57. Véase asimismo G. Singer, "Die Kontroverse zwischen Alexander Friedmann und Albert Einstein um die Möglichkeit einer nichtstatischen Welt", en H. W. Duerbeck y W. R. Dick (eds.), Einsteins Kosmos, op. cit., pp. 142-61.

${ }^{50}$ Un breve repaso de la vida y aportaciones científicas de Georges Lemaître, en J.-P. Luminet, L'invention du Big Bang, op. cit., cap. 8.

${ }^{51}$ Así lo resume J. Cepa: "Vesto Slipher midió por primera vez, entre 1912 y 1925, líneas de emisión en los espectros de 40 'objetos nebulares'. Sorprendido, constató que [las] líneas espectrales [...] se habían 'desplazado al rojo'. Este fenómeno afectaba a todos los objetos medidos en aquellos años, salvo la nebulosa de Andrómeda. Por aquel entonces, ni siquiera estaba establecida la naturaleza de dichos objetos nebulares como galaxias similares a la nuestra. Los 'objetos nebulares' o 'nebulosas' constituían una categoría que englobaba una gran variedad de objetos tanto galácticos, consistentes en nebulosas de emisión o reflexión, planetarias, bipolares, regiones HII, etc.; como extragalácticos, consistentes en galaxias de todas las clases y tipos morfológicos. Los objetos nebulares que estudió Slipher pertenecían al tipo de 'nebulosas espirales', es decir, lo que en la actualidad conocemos como galaxias espirales. Posteriormente, Edwin Hubble y Milton Humason (personaje singular que pasó de ser el mulero que transportaba material y suministros a Mount Wilson a ser el portero del observatorio, luego el asistente de Hubble y finalmente su colaborador) extendieron la lista de Slipher a más galaxias pertenecientes a distintos cúmulos, y encontraron que no existía ninguna cuyo espectro estuviera desplazado al azul". J. Cepa, Cosmología física, op. cit., pp. 33-4. Las observaciones astronómicas de Hubble, llevadas a cabo durante los años 1922 y 1924, le permitieron probar que las nebulosas espirales eran en realidad galaxias enteras independientes de la Vía Láctea y que estaban compuestas por millones de estrellas:
} 
espirales $^{52}$. Para Lemaître la velocidad de recesión de las "nebulosas extragalácticas" no era sino el indicio de un universo en expansión ${ }^{53}$. En estos términos ha explicado Stephen Hawking la encrucijada del momento:

"En esa época casi todos esperaban que las galaxias se estuvieran moviendo de forma más bien aleatoria, y en consecuencia esperaban encontrar tantos espectros desplazados al azul como desplazados hacia el rojo. Por eso fue una sorpresa descubrir que todas las galaxias aparecían desplazadas hacia el rojo. Cada una de ellas se estaba alejando de nosotros. Todavía más sorprendente era el resultado que Hubble publicó en 1929: tampoco el tamaño del desplazamiento hacia el rojo de las galaxias era aleatorio, sino que era directamente proporcional a la distancia de la galaxia a nosotros; o, en otras palabras, cuanto más lejos estaba la galaxia, con más rapidez se alejaba. Eso significaba que el universo no podía ser estático, como se creía hasta entonces, sino que en realidad se estaba expandiendo. La distancia entre las diferentes galaxias aumentaba continuamente" ${ }^{5_{4}}$.

Los sucesivos periodos de investigación de Lemaître en Inglaterra -en 1923, donde pudo colaborar estrechamente con Arthur Eddington en Cambridge- y en los Estados Unidos - en 1924, donde tuvo oportunidad de trabajar con Harlow Shapley y conocer de primera mano los resultados preliminares de los trabajos de Vesto Slipher y Edwin Hubble en el Massachusetts Institute of Technology $(M I T)^{55}$ - le permitieron preparar el marco teórico de interpretación en el que los nuevos hallazgos observacionales adquirirían una inusitada significación cosmológica... abominable desde el punto de vista de Einstein.

\footnotetext{
"Los trabajos de Hubble abrían decididamente la puerta a un gigantesco universo poblado de nebulosas a modo de 'otras vías lácteas', a miles de millones de años luz. Los componentes de ese universo ya no eran estrellas sino 'galaxias', tal como Shapley propuso denominar a las nebulosas extragalácticas". A. Rioja y J. Ordóñez, Teorías del universo, Madrid, Editorial Síntesis, vol. 3, 2006, p. 328. Muy oportunamente, A. Rioja y J. Ordóñez señalan "que hay una frontera que distigue y separa el estudio del universo de las estrellas del de las galaxias". En este sentido, "al contemplar todo lo acaecido en astronomía en las últimas décadas del siglo XIX y primeras del siglo xx no es de extrañar que se haya hablado de una 'segunda revolución astronómica' por contraposición a la que se iniciara con la obra de Copérnico en el siglo xvI". Ibidem, pp. 326 y 332.

${ }^{52}$ Lemaître tuvo oportunidad de asistir a la famosa reunión de Washington de finales de 1924 en la que Edwin Hubble demostró que la Vía Láctea no era la única galaxia existente. Para más detalles véase J.P. Luminet, L'invention, p. 89: “À la fin de 1924, [Lemaître] assiste à une réunion à Washington restée célèbre, puisque y est annoncée la découverte de Céphéides par Edwin Hubble dans les nébuleuses spirales, grâce à l'utilisation du gran télescope du mont Wilson. Cela permet de prouver l'existence de galaxies extérieures à la nôtre. Lemaître comprend aussitôt que cette nouvelle conception des 'Universîles' aura des conséquences pour les théories de la cosmologie relativiste".

${ }_{53}^{5}$ J.-P. Luminet, L'invention du Big Bang, op. cit., pp. 90-1 y 101-2.

${ }^{54}$ S. W. Hawking, La teoría del todo. El origen y el destino del Universo [1994], trad. esp. de J. García Sanz, Barcelona, Debolsillo, 2010, p. 30.

${ }^{55}$ J.-P. Luminet, L'invention du Big Bang, op. cit., pp. 87-9.
} 


\section{Misterios abominables: la expansión del universo y la reocupación cosmológica de la creatio ex nihilo}

Apenas sí alcanzamos a imaginar la extrañeza que el joven físico George Lemaître, ataviado con su hábito de sacerdote católico, pudo haber causado en Einstein en el momento de su primer encuentro ${ }^{56}$. Éste se produjo en Bruselas, en 1927, ciudad a la que Einstein había viajado con motivo del Quinto Congreso Solvay, dedicado a la mecánica cuántica. Lemaître recordó el encuentro tardíamente en una locución de radio grabada en conmemoración de los dos años de la muerte de Einstein $^{57}$ : "paseándose por el parque Leopold, me hablaba de un artículo, poco conocido, que yo mismo había escrito el año precedente sobre la expansión del universo ${ }^{58}$, y que un amigo le había recomendado ${ }^{59}$. Tras algunos comentarios técnicos favorables, concluyó que, desde el punto de vista de la física, todo aquello le resultaba abominable ['tout à fait abominable']" 60 .

A pesar del inesperado exabrupto, Lemaître no pudo sino agradecer a Einstein que le diera noticia de los trabajos de Friedmann ${ }^{61}$, que por entonces todavía ignoraba ${ }^{62}$. Dado que parecía deseoso de continuar la conversación, el acompañante de Einstein, Auguste Piccard, invitó a Lemaître a que se fuera con ellos en el taxi, pues tenían previsto visitar los laboratorios de la Universidad de Bruselas. Según Lemaître, en el taxi pudo hablarle a Einstein "de las velocidades de las nebulosas

\footnotetext{
${ }^{56}$ K. Roessler, "Georges Lemaître, das expandierende Universum und die kosmologische Konstante", en H. W. Duerbeck y W. R. Dick (eds.), Einsteins Kosmos, op. cit., p. 169: "Die hier gezeigte Momentaufnahme ist bezeichnend für Einsteins gelegentlich selbstherrliche Attitüde und sein misstrauisches Staunen über einen jungen Physiker im Habit eines katholischen priesters, selbst wenn er ihm die mathematische Beherrschung der allgemeinen Relativitätstheorie bescheinigen musste. Auch Eddington, der Lemaître von seinem Gastaufenthalt in Cambridge im Jahr 1923 viel besser kannte, äusserte zunächst das Verdikt des 'Theologischen' über dessen Ansatz”. Para una descripción del encuentro se puede ver J.-P. Luminet, L'invention du Big Bang, op. cit., pp. 102 y ss. Véase también O. Godart y M. Heller, "Einstein-Lemaître: Recontre d'idées", Revue des Questions Scientifiques 150, 1979, pp. 23-43; D. Lambert, Un atome d'univers. La vie et l'oevre de Georges Lemaître [2000], Bruxelles, Éditions Lessius, 2011.

${ }^{57}$ K. Roessler, ibidem, p. 168.

${ }^{58}$ Parece que Lemaître se refería a su artículo "Un Univers homogène de masse constante et de rayon croissant, rendant compte de la vitesse radiale des nébuleuses extra-galactiques", en Annales de la Societé scientifique de Bruxelles, série A, t. XLVII, avril 1927, pp. 29-39 (49-59).

${ }^{59} \mathrm{Se}$ cree que este amigo podría ser Théophile De Donder, con quien Einstein colaboró en Bruselas en la preparación del Quinto Congreso Solvay, precisamente. Véase K. Roessler, ibidem, pp. 168-9.

${ }^{60}$ Citado por J.-P. Luminet, L'invention du Big Bang, op. cit., p. 103; también por K. Roessler, "Georges Lemaître, das expandierende Universum und die kosmologische Konstante”, op. cit., p. 168.

${ }^{61}$ Que el propio Einstein había reseñado en dos ocasiones: A. Einstein, "Bemerkung zu der Arbeit von A. Friedmann: Über die Krümmung des Raumes", Zeitschfrift für Physik 11, 1922, p. 326; "Notiz zu der Arbeit von A. Friedmann: Über die Krümmung des Raumes", en Zeitschfrift für Physik 16, 1923, p. 228.

${ }^{62}$ J.-P. Luminet, L'invention du Big Bang, op. cit., p. 101.
} 
extragalácticas", pero "tenía la impresión de que [Einstein] no estaba al corriente de los hechos astronómicos" $"$.

La ulterior "conversión" de Einstein a un modelo no estático de universo, esto es, la rotunda aceptación de las implicaciones físicas y aún teológicas de un cosmos en expansión, hubo de producirse algunos años después, cuando Einstein viajó a California ${ }^{64}$ y tuvo oportunidad de conocer el California Institute of Technology, el Mount Wilson Observatory y encontrarse con Edwin Hubble y Richard Chace Tolman [1881-1948], entre otros astrónomos americanos ${ }^{65}$ Fue entonces cuando vió con sus propios ojos el desplazamiento al rojo de las nebulosas espirales extragalácticas, y no pudo sino aceptar la expansión del universo y renunciar a la constante cosmológica $^{66}$.

Einstein propuso entonces dos modelos de universo en expansión, el denominado "universo de Friedmann-Einstein"67 -en 1931- y el "universo de Einstein-De Sitter" 68 -en 1932, en un artículo escrito en coautoría con el matemático y astrónomo holandés Willem de Sitter-. En ambos casos, se asumía un modelo de universo espacialmente cerrado pero desprovisto de constante cosmológica ${ }^{69}$.

\footnotetext{
${ }^{63}$ Citado por J.-P. Luminet, L'invention du Big Bang, op. cit., p. 103. Luminet añade lo siguiente: "[...] André Deprit (ancien élève de Lemaître) donne une version plus pittoresque et légèrement différente de cette rencontre. Il affirme notamment que Lemaître ne connaissait pas l'allemand, ce qui peut expliquer le fait que le savant belge n'ait pas cité le travail antérieur de Friedmann das son article de 1927".

${ }^{64}$ No sin antes pasar por La Habana... Véase A. Marqués Dols, "Albert Einstein: treinta horas en La Habana", en Desde Cuba, 7 de julio de 2011.

${ }^{65}$ Así se lo relataba Einstein a su amigo Michele Besso: "Die Leute vom Mount Wilson-Observatorium sind ausgezeichnet. Sie haben in letzter Zeit gefunden, dass die Spiralnebel räumlich annähernd gleichmäßig verteilt sind und einen ihrer Distanz proportionalen mächtigen Dopplereffekt zeigen, der sich übrigens aus der Relativitätstheorie zwanglos folgern lässt (ohne kosmologisches Glied). Der Haken ist aber, dass die Expansion der Materie auf einen zeitlichen Anfang schliessen lässt, der $10^{10}$, bezw. $10^{11}$ Jahre zurückliegt", en P. Speziali, Albert Einstein-Michele Besso: Correspondence 19031955, A. Hermann, Paris, p. 268, citado por T. Jung, "Einsteins Beitrag zur Kosmologie - ein Überblick", en H. W. Duerbeck y W. R. Dick (eds.), Einsteins Kosmos, op. cit., p. 88.

${ }^{66}$ T. Jung, ibidem, pp. 84-95. Por entonces Arthur Eddington demostraría que el universo de Einstein se encontraba en equilibrio inestable: A. S. Eddington, "On the instability of Einstein's spherical World", Monthly Notices of the Royal Astronomical Society 90, 1930, pp. 668-78.

${ }^{67}$ A. Einstein, "Zum kosmologischen Problem der allgemeinen Relativitätstheorie", Sitzungber. Preuß. Akad. Wiss., 96, 1931, pp. 235-237.

${ }^{68}$ A. Einstein y W. de Sitter, "On the relation between the expansion and the mean density of the universe", en Proceedings of the National Academy of Sciences of the United States of America 18, 1932, pp. 213-4. Las aportaciones de Einstein también dieron lugar a los así conocidos como modelos Einstein-Weyl. En estos modelos se trataba de preservar un universo estático y explicar, al mismo tiempo, el fenómeno del desplazamiento al rojo. Véase, por ejemplo, E. Scholz, "Einstein-Weyl Models of Cosmology", en J. Renn (ed.), en Albert Einstein. Chief Engineer of the Universe. Einstein's Life and Work in Context, Berlin, WILEY-VCH, 2005, pp. 394-7.

${ }^{69}$ T. Jung, op. cit., p. 68.
} 
Es por todo ello que el segundo encuentro entre Einstein y Lemaître ${ }^{70}$, en Pasadena, en 1933, había de poseer una transcendencia especial para ambos. No sólo porque Lemaître había tenido razón sobre Einstein -a pesar de la fuerte oposición de este último ${ }^{71}-$, ni siquiera porque para entonces ya gozase de una reputación bien establecida $^{72}$, sino porque la abominable reocupación cosmológica de la escatología se había consumado y portaba ya las credenciales de una ciencia de vanguardia. Más aún: Lemaître la había llevado un poco más lejos con su audaz hipótesis del átomo primitivo ${ }^{73}$ : "en lugar de considerar el mundo estático de Einstein como un estado inicial desde el cual concebir el modelo dinámico, Lemaitre había preferido pensar que el Universo comenzó su expansión a partir de un estado singular" ${ }^{\prime 74}$. De acuerdo con Lemaître, además de expandirse, el universo poseía un origen designable y excepcional.

En Pasadena, Einstein no se encontró "con un joven y desconocido científico cualquiera, sino con un hombre cuyas ideas habían dado mucho que hablar, [...] promotor de un modelo cosmológico capaz de predecir la ley de Hubble"75. El 11 de enero de 1933, Einstein asistió a un seminario que Lemaître estaba impartiendo en esa ciudad californiana, y que en cierto modo suponía el reconocimiento oficial de su teoría por parte de la comunidad científica ${ }^{76}$. Lemaître aprovechó la ocasión para exponer su idea del nacimiento del universo a partir de un "átomo primitivo", tal como había sugerido recientemente en sus tres artículos "The expanding Universe", "The beginning of the World from the point of view of quantum theory" $y$ "L'expansion de l'espace", todos ellos aparecidos en $1931^{77}$. En su opinión, el comienzo del universo había sido muy diferente respecto a su estado actual. Si a día de hoy el universo se encuentra en expansión, ello se debe a que en el pasado era inmensamente más denso

\footnotetext{
${ }^{70}$ Para los ulteriores encuentros entre Einstein y Lemaître véase K. Roessler, "Georges Lemaître, das expandierende Universum und die kosmologische Konstante", op. cit., pp. 172 y ss.

${ }^{71}$ K. Roessler, ibidem, p. 162.

${ }^{72}$ J.-P. Luminet, L'invention du Big Bang, op. cit., p. 138.

${ }^{73}$ Así la definió el propio Lemaître en 1945: "L'hypothèse de l'atome primitif est un hypothèse cosmogonique suivant laquelle le monde actuel a résulté de la désintégration radiactive d'un atome". G. Lemaître, "L'hypothèse de l'atome primitif", Actes de la Société helvétique des sciences naturelles, 1945, pp. 77-96, recogido en J.-P. Luminet, L'invention du Big Bang, op. cit., pp. 203-226.

${ }^{74}$ Ibidem, p. 109.

${ }^{75}$ Ibidem, pp. 138-9.

${ }^{76} \mathrm{~K}$. Roessler, op. cit., p. 170.

${ }^{77}$ El primero de ellos, de carácter técnico, apareció en marzo de 1931 en Monthly Notices of the Royal Astronomical Society (91, 1931, pp. 490-501); mientras que el segundo y el tercero eran versiones divulgativas simplicadas, publicadas en Nature $(127, \mathrm{p} .706)$ y en la Revue des questions scientifiques (50 année, $4^{\mathrm{e}}$ série, t. XX, 1931, pp. 391-410) en mayo y noviembre de ese mismo año, respectivamente. Para más detalles véase J.-P. Luminet, L'invention du Big Bang, op. cit., pp. 111 y ss.
} 
y condensado ${ }^{78}$, una suerte de quantum único ${ }^{79} \mathrm{o}$ núcleo atómico gigantesco ${ }^{80}$, cuya sucesiva desintegración ${ }^{81}$ y ulterior fragmentación dio inicio a la expansión del universo. De este modo, "las nociones de espacio y tiempo no tendrían significado en el comienzo, sino que adquirieron algún sentido progresivamente, cuando el quantum original se dividió [...] en un número suficiente de quanta parciales" ${ }^{22}$. A saber, "el comienzo del mundo tuvo lugar un poco antes que el del espacio y el tiempo" 83 , y era a partir de ese momento que la historia del universo podía "ser escrita etapa por etapa" ${ }^{44}$.

Así planteadas las cosas, la protesta de Einstein no se hizo de esperar. La hipótesis del átomo primitivo no sólo le parecía insostenible desde el punto de vista de la física, sino una conjetura inverosímil inspirada por la doctrina cristiana de la creación: "Nein, nicht so etwas. Das erinnert zu sehr an die Schöpfungslehre!" Einstein se negó en redondo a discutir sobre la hipótesis del átomo primitivo, pues consideraba que en este punto -como en tantos otros- el sacerdote belga no estaba siendo científicamente objetivo ${ }^{86}$ y se había dejado llevar por los dogmas de la teología cristiana. Pero los desarrollos en astrofísica y cosmología producidos durante las décadas siguientes, demostraron - una vez más- que Einstein se equivocaba y que Lemaître tenía razón. La execrable reocupación cosmológica de la creatio ex nihilo dio lugar a la teoría del Big Bang y, ulteriormente, al llamado "modelo estándar de la cosmología contemporánea" ${ }^{" 87}$.

\footnotetext{
${ }^{78}$ J.-P. Luminet, L'invention du Big Bang, op. cit., pp. 90-1; A. Rioja y J. Ordóñez, Teorías del universo, op. cit., pp. 341-7.

${ }^{79}$ G. Lemaître, "L'origine du monde du point de vue de la théorie quantique" [1931] -título original: "The beginning of the World from the point of view of quantum theory"-, recogido en J.-P. Luminet, L'invention du Big Bang, op. cit., p. 129.

${ }^{80}$ É. Klein, Discours sur l'origine de l'univers, Paris, Flammarion, 2010, p. 36, nota 2.

${ }^{81}$ Lemaître, "L'origine du monde du point de vue de la théorie quantique", op. cit., p. 129.

82 "Si le monde a commencé par un quantum unique, les notions d'espace et de temps n'auront absolument plus de signification au commencement même; mais elles acquerront progressivement quelque sens, quand e quantum originel se sera divisé [...] un nombre suffisant de quanta partiels". Lemaitre, ibidem.

83 “[...] le début du monde a [eu] lieu un peu avant celui de l'espace et du temps". Lemaître, ibidem.

84 “'.... [il n'est pas nécessaire que l'histoire entière de l'Univers ait été inscrite dans le premier quantum, comme une mélodie sur le disque d'un phonographe. La totalité de la matière de l'Univers doit été présente dès commencement, mais l'histoire qu'elle nous raconte peut être écrite étape par étape". Ibidem.

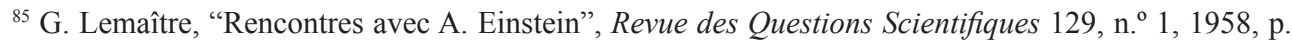
130, citado por K. Roessler, op. cit., p. 171. Véase también J.-P. Luminet, L'invention du Big Bang, op. cit., p. 139

${ }^{86}$ J.-P. Luminet, L'invention du Big Bang, op. cit., p. 139.

${ }^{87}$ J. Cepa, Cosmología física, op. cit. pp. 176-7; E. Scholz, "The Standard Model of Contemporary Cosmology", en J. Renn (ed.), Albert Einstein. Chief Engineer of the Universe, op. cit., pp. 388-93. De este modo lo explican A. Rioja y J. Ordóñez: "La cuestión de la expansión del universo y de la recesión (isotrópica) de las galaxias conduce a plantearse la sucesiva evolución del estado del universo en un tiempo
} 


\section{Escatología y apocalipsis en perspectiva cosmológica}

Hasta aquí hemos intentado mostrar que la dimensión escatológica de la astronomía contemporánea no se limita a una cualidad accesoria de algunas de sus especulaciones más aventuradas, sino que es una configuración interna, históricamente constituida, de su orden disciplinar y epistemológico. Es por ello -y por razones de claridad- que creemos conveniente distinguir entre cosmología escatológica y apocalipsis cosmológico. Por la primera entendemos la reocupación cosmológica de la escatología, que se especifica en la producción de una cosmogonía mítico-cientifica encaminada a describir y explicar el origen del tiempo cósmico; así como en la formulación de una promesa escatológica, que bajo la forma de la predicción científica augura que el final del universo habrá de llegar de manera necesaria.

Reservamos, en cambio, la noción de apocalipsis cosmológico para referirnos a las representaciones mítico-científicas del final del universo, incluyendo también los eventos cósmicos excepcionales -de índole cataclísmica- que puedan afectar a algunas regiones del universo conocido, previamente singularizadas por su significación para el hombre. En este sentido, cabría diferenciar a su vez entre un apocalipsis cosmológico a pequeña escala (o apocalipsis vicario) y un apocalipsis cosmológico a gran escala, si bien este último significado habría de prevalecer sobre el primero cuando se hace referencia al apocalipsis cosmológico en cuanto tal.

A nuestro juicio, tanto la cosmología escatológica como el apocalipsis cosmológico habrían practicado a su vez una reocupación (astronómica) del mito y de la metáfora. Los hallazgos en astronomía observacional y en astrofísica extragaláctica producidos durante los siglos Xx y XxI, así como la subsiguiente multiplicación de especialidades y subespecialidades que componen en la actualidad la ciencia astronómica -como la radioastronomía, la geología planetaria, la astronometría o la astronomía de rayos $\mathrm{X}$, entre otras muchas-, no sólo han permitido la pervivencia del mito sino que han propiciado su secreta renovación. Con sus sensacionales descubrimientos, la astronomía contemporánea ha remitificado el cosmos, produciendo de paso nuevos mitos del origen y del final del universo, en clara consonancia con la tradición cosmogónica en la que de hecho se inscribe.

La astronomía contemporánea ha extendido, entre sus mitos y metáforas del origen y del final del tiempo cósmico, una densa metafórica organicista y biologicista

anterior. En efecto, si retrocedemos hacia el pasado, necesariamente nos vemos conducidos a estados de mayor densidad, en los que el espacio interestelar e intergaláctivo hubo de ser progresivamente menor hasta alcanzar la materia un estado primero de densidad infinita (punto de curvatura infinita del espacio). Se trata de lo que se conoce con el nombre de 'singularidad' (en sentido matemático) inicial. Cualquier intento de explicar científicamente el origen del universo pasa por describir el modo en que galaxias y estrellas han podido constituirse a partir de ciertas condiciones iniciales. En definitiva, nos vemos abocados a algún tipo de 'gran explosión' originaria, a partir de la cual todo se habría formado". A. Rioja y J. Ordóñez, Teorías del universo, op. cit., pp. 340-1. 
con la que dar cuenta de la formación y evolución del universo. La metáfora dominante de las edades del universo ha permitido articular el "pathos escatológico" ["eschatologische Pathos"] ${ }^{88}$ de la cosmología física contemporánea, y hacer de sus "visiones apocalípticas" [“apokaliptischen Visionen"] ${ }^{89}$ el contenido de una fenomenología escatológica del $\operatorname{cosmos}^{90}$. Con ello se demuestra, una vez más, la necesidad humana de poseer una imagen del hundimiento de todas las $\operatorname{cosas}^{91}$.

\footnotetext{
${ }^{88}$ H. Blumenberg, Vollzähligkeit der Sterne [1997], Frankfurt am Main, Suhrkamp Verlag, 2000, p. 32 ${ }^{89}$ Ibidem, p. 253.

${ }^{90}$ Tomo la expresión "fenomenología escatológica" ["phänomenologische Eschatologie"] de P. Stoellger, "Über die Grenzen der Metaphorologie. Zur Kritik der Metaphorologie Hans Blumenbergs und den Perspektiven ihrer Fortschreibung", en A. Haverkamp y D. Mende (eds.), Metaphorologie. Zur Praxis von Theorie, Frankfurt am Main, Suhrkamp Verlag, 2009, p. 226.

91 "Das Bedürfnis nach Untergangsvisionen, nach dem Erschrecken vor ihnen und mit ihnen, erwies sich als unausrottbar". H. Blumenberg, Vollzähligkeit der Sterne [1997], Frankfurt am Main, Suhrkamp Verlag, 2000, p. 251.
} 